\title{
Personalidad y clima social familiar en adolescentes de familia nuclear, biparental y monoparental
}

\author{
Personality and family social climate in adolescents of nuclear, biparent \\ and single-parent family
}

\author{
Silvia Chuquimajo Huamantumba ${ }^{1}$ \\ Universidad Privada del Norte
}

Recibido: $23-10-17$

Aceptado: $21-11-17$

\begin{abstract}
Resumen
La presente investigación plantea analizar las diferencias entre la personalidad y el clima social familiar en adolescentes que pertenecen a familia nuclear biparental y monoparental. La muestra estuvo conformada por 254 adolescentes de entre 13 y 19 años de instituciones educativas estatales de Lima Metropolitana. Se aplicó el Inventario de Personalidad para Adolescentes (MAPI) de Millon y la Escala de Clima Social en la Familia (FES) adaptada por Ruiz y Guerra. El diseño es descriptivo comparativo. Los resultados muestran que el tipo de familia (nuclear biparental y monoparental) no influye en los Estilos Básicos de Personalidad, no obstante, si hay influencia del sexo sobre la personalidad. Se concluye que el tipo de familia si influencia sobre la personalidad, pero no sobre el clima familiar, el tipo de familia de la que se forma parte, no es por sí misma una variable relevante para influir en el clima social familiar.

Palabras clave: Familia monoparental; familia nuclear biparental; personalidad; clima social familiar; adolescentes.
\end{abstract}

\begin{abstract}
This research raises analyze the differences in the personality and the family social climate of adolescents between who belong to nuclear parent Family and single-parent family. The sample consisted of 254 adolescents between 13 and 19 years of State educational institutions of Metropolitan Lima. We administered the Inventory of adolescents' personality (MAPI) and the scale of family social climate (FES) adapted by Ruiz and Guerra. The design is descriptive comparative. The results show that the type of family (nuclear parent family and single- parent family) does not influences in the styles of personality, but is influences by the sex. The social family climate and type of family does not show differences significant in the
\end{abstract}

1 Universidad Privada del Norte. silviachuquimajo@outlook.com

(C) Los autores. Este artículo es publicado por la Revista de Investigación en Psicología de la Facultad de Psicología, Universidad Nacional Mayor de San Marcos. Este es un artículo de acceso abierto, distribuido bajo los términos de la licencia Creative Commons Atribucion - No Comercia_Compartir Igual 4.0 Internacional. (http://creativecommons.org/licenses/by-nc-sa/4.0/) que permite el uso no comercial, distribución y reproducción en cualquier medio, siempre que la obra original sea debidamente citada. 
scale, neither in the dimensions. In conclusion, the type of family influence in the personality but not in the social family climate; the type of family that the adolescent belong is not the only relevant variable to affect in the social family climate.

Key words: single-parent family; nuclear parent family; personality; social climate family; adolescents.

Los padres como soporte socio afectivo de los hijos moldean la personalidad y generan un clima particular en la familia, no obstante, observamos en el país el incremento de familias monoparentales, conformada generalmente la madre. Por ello nuestro problema de investigación se formula de la siguiente manera ¿Existirán diferencias significativas en la Personalidad y el Clima Social Familiar en adolescentes de ambos sexos de Familia Nuclear Biparental y Monoparental? El objetivo general de la investigación es determinar las diferencias en la Personalidad y el Clima Social Familiar en adolescentes de ambos sexos de Familia Nuclear Biparental y Monoparental.

En relación a la personalidad tomamos como referencia la teoría de Theodore Millon, quien desarrolló la teoría del aprendizaje biosocial y la teoría evolutiva (Millon, 1969, citado por Heim \& Westen, 2007). De acuerdo a Cardenal, Sánchez y Ortiz-Tallo (2007) los postulados de Millon se caracterizan por: a) El uso de una perspectiva teórica integradora. Integra la perspectiva nomotética, que permite descubrir cómo se relacionan las necesidades, los motivos, los mecanismos, los rasgos, los esquemas, etc., e ideográfico, que comprende que la personalidad es el resultado de una historia única de transacciones de factores biológicos y contextuales. b) comprende que lo normal y patológico son conceptos relativos, típico dentro de un continuo, no como categorías nominales. La personalidad normal es entendida como los estilos de adaptación que resultan eficaces en entornos normales, no obstante, los trastornos son comprendido como estilos de funcionamiento inadaptados que pueden atribuirse a deficiencias, desequilibrios o conflictos en la capacidad para relacionarse con lo habitual. c) La inclusión de principios de la Teoría de la Evolución para analizar la estructura y la dinámica de los Estilos de Personalidad.

Con respecto al clima social familiar, tomamos los planteamientos de Moss (1974, citado por Zavala 2001) señala que el entorno es un factor importante para el bienestar, el soporte del comportamiento humano, debido a que contempla variables sociales y físicos que influyen en el desarrollo del individuo. Para el autor el clima social familiar es descrito en tres dimensiones (citado en Jiménez, Fernández y Godoy, 2000):

Dimensión Relación: mide la avenencia y ayuda entre los miembros de la familia, así como la expresión de sentimientos, tanto positivos como negativos (Billings \& Moos, 1981). Esta dimensión comprende tres áreas: a) Cohesión: grado en que los integrantes de la familia se comprenden y se sostienen entre sí. b) 
Expresividad: grado en el que se fomenta la libre comunicación de sentimientos. c) Conflicto: nivel en el que comunican claramente su molestia y problema.

Dimensión Desarrollo: Implica conocer el valor de ciertos procesos de crecimiento personal, como los aspectos culturales, políticos, sociales, recreativos entre otros (en Calderón y De la Torre, 2005). Esta dimensión comprende las áreas: a) Autonomía: nivel potestad para decidir libremente. b) Actuación: nivel en que las actividades académicas o laboral se encuadran en una estructura enfocada a la acción. c) Intelectual - Cultural: disposición a actividades de tipo políticointelectual, colectivo y cultural. d) Social-Recreativo: participación en actividades de esparcimiento. e) Moralidad Religiosidad: interés a los valores ético y religioso.

Dimensión Estabilidad: Se entiende al ordenamiento familiar y el grado de inspección que ejercen los miembros entre sí (en Calderón y De la Torre, 2005). Esta dimensión comprende las siguientes áreas: a) Organización: consideración e interés para planear actividades y el compromiso que ello concierne. b) Control: empleo de reglas y procedimientos establecidos en la convivencia familiar.

Podemos agregar que existen diversas investigaciones relacionadas a la variable del estudio, tales como:

Clemens (1988) exploró la depresión y la agresión en un grupo de adolescentes varones de 12 a 14 años, institucionalizados en un hogar transitorio del Callao. El estudio señala que la familia disgregada y desintegrada es un factor que influye en los sentimientos de agresión del adolescente además encontró un alto porcentaje de signos de agresión y depresión en jóvenes donde se apreciaba la ausencia de la figura paterna o de ambos padres.

McLanahan \& Sandefur (en Chouhy, 2001) estudiaron la función paterna y la familia monoparental en adolescentes y adultos jóvenes de ambos sexos. Encontraron un 50\% mayor de riesgo a postergar los estudios y trabajo por periodos prolongados y un $100 \%$ más alto de interrumpir estudios secundarios y de embarazo en jóvenes que crecieron sin su padre.

Perales y Sogui (1997) analizaron las variables socio familiares, personales y contextuales con conductas violentas accidentales o intencionales en adolescentes de 12 a 17 años de edad residentes de Lima. Identificaron como factor de riesgo el sexo masculino, la edad entre 15 y 17 años, la estructura familiar desintegrada, mayor densidad habitacional y tendencia antisocial.

Jiménez, Fernández y Godoy (2000) estudiaron la correspondencia del clima familiar con los hijos de padres separados y que permanecían juntos. Los resultados no muestran diferencias significativas en la variable clima familiar, con salvedad de la variable moralidad-religiosidad, siendo superior en las familias con ambos padres. 
Pichardo, Fernández de Haro y Amezcua (2002) analizan la importancia del clima social familiar en la adaptación personal y social de los adolescentes de ambos sexos. Los reportes señalan que los adolescentes que perciben un clima familiar como elevado en cohesión, expresividad, organización, participación en actividades intelectuales y con un nivel de interés por valores de índole ético y religioso y con niveles bajos en conflicto, muestran mejor adaptación general que los adolescentes cuyas percepciones van en dirección inversa.

Commanor \& Phillips (2002) estudiaron el impacto de los ingresos familiares y diversas dimensiones de la estructura familiar en el contacto con el sistema de justicia penal en jóvenes de 14 y 22. Los resultados indican que la ausencia del padre y la delincuencia juvenil y violencia hay una fuerte relación; este problema se complejiza en varones criados sin figura paterna.

Salazar (2003) analizó el clima social en la familia y los relacionó con los tipos de familia en mujeres adolescentes en condición de gestantes. No halló relaciones significativas entre las dimensiones del clima social familiar y los tipos de familia según su composición. Concluye que el tipo de familia no es una variable que determina la forma de interrelación familiar.

Wilson (1994, en Kliksberg, 2004) reporta el estudio realizado en 60.000 niños. Señala que, independiente del ingreso económico, salvo el muy alto, en ambos sexos, blancos, negros e hispanos, los niños que vivían con una madre divorciada o soltera en comparación con los niños que vivían con ambos padres biológicos, eran doblemente proclives a ser retirados de la escuela, a presentar problemas emocionales o de conducta y de relación con sus compañeros de escuela.

Rosales y Espinoza (2009) analizaron la percepción del clima familiar de adolescentes que pertenecen a diferentes tipos de familia. Los estudios señalan que, si el clima familiar es adecuado, independientemente que el tipo de familia, se puede proveer a sus miembros de las condiciones adecuadas para su desarrollo y supervivencia.

Paz-Navarro, Rodríguez y Martínez (2009) evaluaron el funcionamiento familiar de estudiantes con bajo rendimiento escolar y rendimiento escolar promedio. Reportan que los estudiantes con bajo rendimiento escolar pertenecían a varios tipos de familia (completa, extensa, reconstruida y monoparental) no obstante, la mayoría de los alumnos que presentaban un rendimiento escolar promedio pertenecían a familia completa.

Frisancho (2011) estudió la violencia en el ámbito escolar y su relación con el Funcionamiento Familiar en adolescentes. Reportó que los adolescentes varones obtienen mayor porcentaje en actitudes favorables hacia la violencia $(31,0 \%)$, intimidación (24\%), conductas agresivas $(25 \%)$, no obstante, las mujeres ejercen con mayor tendencia conductas pro sociales $(28,0 \%)$. Otro adato importante es 
que la mayoría de ellos pertenece a familias con características disfuncional o severamente disfuncional.

Después de la revisión teórica y de la investigación de variables estudiadas, postulamos que la personalidad de los adolescentes guarda relación con la composición familiar, los diferentes estudios explican que en las familias integradas por un solo padre los hijos muestran signos de agresión física, tendencia al resentimiento, sentimiento interno de abandono, soledad, disminuida autoestima, niveles de estrés elevado, disminuido rendimiento escolar, con tendencia a ser retirados o suspendidos del ámbito escolar, a interrumpir sus estudios, a tener dificultades con sus compañeros y proclives a tener una conducta antisocial, en comparación con los adolescentes que viven con ambos padres que se desarrollan sin mucho coste cognitivo-emocional. Con respecto al clima social familiar se ha encontrado resultados disimiles, que indican que los adolescentes cuyo clima familiar es percibido como elevado denotan una mayor adaptación general que sus iguales cuyas percepciones sobre la familia van en dirección inversa, otros estudios no revelan diferencias significativas. Por todo lo mencionado, plantearnos nuestra hipótesis general. Existen diferencias significativas en la Personalidad y el Clima Social Familiar en adolescentes de Familia Nuclear Biparental y Monoparental. Como hipótesis específicas: H1 Existen diferencias significativas en los Estilos Básicos de Personalidad en adolescentes de Familia Nuclear Biparental y Monoparental. H2 Existe diferencias significativas en los Estilos Básicos de Personalidad según el sexo de los adolescentes. H3 Existen diferencias significativas en los Sentimientos y Actitudes en adolescentes de Familia Nuclear Biparental y Monoparental. H4 Existen diferencias significativas en los Sentimientos y Actitudes según el sexo de los adolescentes. H5 Existen diferencias significativas en el aspecto Comportamental en adolescentes de Familia Nuclear Biparental y Monoparental. H6 Existen diferencias significativas en el aspecto Comportamental según el sexo de los adolescentes. H7 Existen diferencias significativas en el Clima Social Familiar en adolescentes de Familia Nuclear Biparental y Monoparental. H8 Existen diferencias significativas en el Clima Social Familiar según el sexo de los adolescentes.

\section{MÉTODO}

El presente estudio se realizó según el método descriptivo comparativo (Sánchez y Reyes, 2006) y el diseño Transversal debido a que se recolectó los datos en un tiempo único, buscando describir las variables de estudio y examinar su incidencia o correspondencia en un tiempo dado (Hernández Fernández y Baptista, 2010). La población estuvo conformada por adolescentes de ambos sexos, entre 13 y 18 años de edad, de $3^{\circ}, 4^{\circ}$ y $5^{\circ}$ de secundaria de instituciones educativas estatales de Lima Metropolitana. La muestra lo conformaron 422 estudiantes (203 varones y 219 mujeres) entre 13 y 18 años de edad, de $3^{\circ}, 4^{\circ}$ y $5^{\circ}$ de secundaria de instituciones educativas estatales de Lima Metropolitana. 
Se utilizó el muestreo no probabilístico de tipo intencionado. Para medir la variable personalidad se utilizó el Inventario de Personalidad para adolescentes de Millón, adaptada al español por Jiménez y Ávila en 1992. Se utilizó la escala Clima Social Familiar Moos, Moos \& Trickett; adaptada por Fernández y Sierra (1984) y normalizada en nuestro país por Ruiz y Guerra (1993).

\section{RESULTADOS}

Al realizar el análisis de los datos se observa que los adolescentes de familia nuclear biparental y monoparental no se diferencian en los Estilos de Personalidad, pero cuando se toma en cuenta el sexo de los mismos se hallan diferencias significativas en la Escala Cooperativo, Violento y Respetuoso (Véase Tabla 1). Con los detalles del párrafo anterior, en referencia a la hipótesis 1 se acepta la hipótesis nula.

Tabla 1

Diferencias en la variable tipo de familia y sexo y la interacción entre ambas, en los Estilos Básicos de Personalidad

\begin{tabular}{llll}
\hline $\begin{array}{l}\text { Escala } \\
\text { Estilos básicos de Personalidad }\end{array}$ & Sexo & $\begin{array}{c}\text { Tipo de } \\
\text { Familia }\end{array}$ & $\begin{array}{c}\text { Sexo }<\text { Tipo } \\
\text { Familia }\end{array}$ \\
\hline Escala 1: Introvertido & $\mathrm{F}=3.06$ & $\mathrm{~F}=0.31$ & $\mathrm{~F}=0.32$ \\
Escala 2: Inhibido & $\mathrm{F}=0.50$ & $\mathrm{~F}=0.66$ & $\mathrm{~F}=0.21$ \\
Escala 3: Cooperativo & $\mathbf{F}=\mathbf{7 . 7 8 ^ { * }}$ & $\mathrm{F}=0.46$ & $\mathrm{~F}=0.69$ \\
Escala 4: Sociable & $\mathrm{F}=0.79$ & $\mathrm{~F}=0.16$ & $\mathrm{~F}=0.53$ \\
Escala 5: Confiado & $\mathrm{F}=0.60$ & $\mathrm{~F}=0.79$ & $\mathrm{~F}=0.04$ \\
Escala 6: Violento & $\mathbf{F}=\mathbf{1 5 . 6 ^ { * * }}$ & $\mathrm{F}=0.07$ & $\mathbf{F}=\mathbf{4 . 6 5 ^ { * }}$ \\
Escala 7: Respetuoso & $\mathbf{F}=\mathbf{4 . 0 3 *}$ & $\mathrm{F}=1.44$ & $\mathrm{~F}=3.12$ \\
Escala 8: Sensible & $\mathrm{F}=0.90$ & $\mathrm{~F}=0.13$ & $\mathrm{~F}=0.94$ \\
\hline
\end{tabular}

Notas: $\mathrm{F}=$ prueba ANOVA. ${ }^{*} \mathrm{p}<0.05 .{ }^{*} \mathrm{p}<0.01$

La ampliación del análisis tomando en cuenta la interacción sexo y tipo de familia sí determina diferencia estadística significativa en la escala Violento (Véase Tabla 2). Los promedios aritméticos en los varones presentan puntajes más elevados que las mujeres en la Escala Violento y las mujeres obtienen valores más alto que los varones en los rasgos Cooperativo y Respetuoso. Tomando en cuenta esta información aceptamos en parte la hipótesis 2 en los Estilos Básicos de la Personalidad, en Cooperativo, Respetuoso y Violento. 
Tabla 2

Estadísticas de las escalas que obtuvieron diferencias significativas según el sexo en los Estilos Básicos de Personalidad

\begin{tabular}{lccccccc}
\hline & \multicolumn{2}{c}{$\begin{array}{c}\text { Varones } \\
(\mathrm{n}=119)\end{array}$} & \multicolumn{2}{c}{$\begin{array}{c}\text { Mujeres } \\
(\mathrm{n}=135)\end{array}$} & \multicolumn{2}{c}{$\begin{array}{c}\text { Diferencias } \\
\text { Estandarizadas }\end{array}$} \\
\cline { 2 - 9 } Escalas Estilos de Personalidad & $\mathrm{M}$ & $\mathrm{DE}$ & $\mathrm{M}$ & $\mathrm{DE}$ & $\mathrm{D}$ & Etiqueta \\
Escala 3: Cooperativo & 17.69 & 3.39 & $\mathbf{1 8 . 6 1}$ & 3.54 & 0.07 & Trivial \\
Escala 6: Violento & $\mathbf{1 6 . 9 9}$ & 3.63 & 15.69 & 3.16 & 0.38 & Pequeña \\
Escala 7: Respetuoso & 20.24 & 3.41 & $\mathbf{2 0 . 8 5}$ & 3.51 & 0.17 & Trivial \\
\hline
\end{tabular}

Notas: $\mathrm{D}=$ Diferencia

De los promedios también se evidencia que los varones de Familia Monoparental presentan valores más elevados que los adolescentes de Familia Nuclear Biparental en la Escala Violento y que las adolescentes mujeres de Familia Monoparental.

Al realizar diferencias en la variable tipo de familia y sexo la interacción entre ambas, en Sentimientos y Actitudes se observa que los adolescentes de familia nuclear biparental y monoparental solamente se diferencian de una manera estadística significativa $(\mathrm{p}<.05)$ en la Escala Inaceptación Sexual; por otro lado, cuando se toma en cuenta el sexo de los mismos se hallan diferencias significativas en Intolerancia Social, Clima Familiar Inadecuado y Desconfianza Escolar. Con lo mencionado, se acepta en parte la hipótesis 3 (Véase Tabla 3).

Tabla 3

Diferencias en la variable tipo de familia y sexo la interacción entre ambas, en Sentimientos y Actitudes

\begin{tabular}{llcc}
\hline \multicolumn{1}{c}{$\begin{array}{c}\text { Escala } \\
\text { Sentimientos y Actitudes }\end{array}$} & Sexo & Tipo de Familia & $\begin{array}{c}\text { Sexo } \\
\text { X Tipo Familia }\end{array}$ \\
\hline Escala A: Deterioro del autoconcepto & $\mathrm{F}=2.84$ & $\mathrm{~F}=0.33$ & $\mathrm{~F}=1.10$ \\
Escala B: Deterioro de la autoestima & $\mathrm{F}=0.43$ & $\mathrm{~F}=0.50$ & $\mathrm{~F}=0.13$ \\
Escala C: Malestar corporal & $\mathbf{F}=2.49$ & $\mathrm{~F}=1.03$ & $\mathrm{~F}=0.15$ \\
Escala D: Inaceptación sexual & $\mathrm{F}=3.6$ & $\mathrm{~F}=4.68^{*}$ & $\mathrm{~F}=0.52$ \\
Escala E: Sentimiento de ser diferente & $\mathrm{F}=1.24$ & $\mathrm{~F}=1.67$ & $\mathrm{~F}=0.01$ \\
Escala F: Intolerancia social & $\mathbf{F}=\mathbf{1 6 . 1}^{* *}$ & $\mathrm{~F}=0.04$ & $\mathrm{~F}=2.55$ \\
Escala G: Clima familiar inadecuado & $\mathbf{F}=\mathbf{5 . 8 ^ { * }}$ & $\mathrm{F}=2.86$ & $\mathbf{F}=\mathbf{5 . 8 6 ^ { * }}$ \\
Escala H: Desconfianza escolar & $\mathbf{F}=\mathbf{1 0 . 2}^{* *}$ & $\mathrm{~F}=0.00$ & $\mathbf{F}=\mathbf{5 . 1 9}^{*}$ \\
\hline
\end{tabular}

Nota: $\mathrm{F}=$ Prueba ANOVA. ${ }^{*} \mathrm{p}<0.05 . * * \mathrm{p}<0.01$

Al realizar la ampliación del análisis de los promedios aritméticos notamos que los varones presentan valores más elevados que las mujeres en la Escala Intolerancia Social, Clima Familiar Inadecuado y Desconfianza Escolar. Con los detalles del párrafo anterior, se acepta en parte la hipótesis 4 (Véase Tabla 4). 
Tabla 4

Estadísticas de las escalas que obtuvieron diferencias significativas en relación al sexo en Sentimientos y Actitudes

\begin{tabular}{lcccccc}
\hline \multirow{2}{*}{$\begin{array}{l}\text { Escala } \\
\text { Sentimientos y Actitudes }\end{array}$} & \multicolumn{2}{c}{$\begin{array}{l}\text { Varones } \\
(\mathbf{n = 1 1 9})\end{array}$} & \multicolumn{2}{c}{$\begin{array}{c}\text { Mujeres } \\
(\mathbf{n = 1 3 5})\end{array}$} & \multicolumn{2}{c}{$\begin{array}{c}\text { Diferencias } \\
\text { Estandarizadas }\end{array}$} \\
\cline { 2 - 8 } & $\mathrm{M}$ & $\mathrm{DE}$ & $\mathrm{M}$ & $\mathrm{DE}$ & $\mathrm{D}$ & Etiqueta \\
Escala F: Intolerancia Social & $\mathbf{9 . 9 1}$ & 3.158 & 8.84 & 2.625 & 0.21 & Pequeña \\
Escala G: Clima familiar Inadecuado & $\mathbf{6 . 9 2}$ & 3.474 & 6.20 & 3.569 & 0.37 & Pequeña \\
Escala H: Desconfianza Escolar & $\mathbf{7 . 8 1}$ & 4.35 & 6.64 & 4.03 & 0.29 & Pequeña \\
\hline
\end{tabular}

Notas: $\mathrm{D}=$ Diferencia.

Al realizar el análisis Estadístico de la interacción tipo de familia y sexo en la Escala Clima Familiar Inadecuado se observa que los promedios aritméticos de los varones de Familia Monoparental son más elevados que los adolescentes de Familia Nuclear Biparental y que las adolescentes mujeres de Familia Monoparental en la Escala Clima Familiar Inadecuado. También notamos que los promedios aritméticos de los varones de Familia Monoparental presentan valores más elevados que las adolescentes de Familia Nuclear Biparental y que las adolescentes mujeres de Familia Monoparental en la Escala Desconfianza Escolar.

Al analizar la variable tipo de familia y sexo y la interacción entre ambas, en el aspecto Comportamental se observa que los adolescentes de familia nuclear biparental y monoparental no se diferencian en este aspecto, pero cuando se toma en cuenta el sexo de los mismos se hallan diferencias significativas en la Escala Disconformidad Social y Rendimiento Escolar Inadecuado. En el análisis donde se toma en cuenta la interacción sexo y tipo de familia se halla diferencia estadística significativa en la escala Disconformidad Social (Véase Tabla 5). Con la información del párrafo anterior, en referencia a la hipótesis 5 , se acepta la hipótesis nula.

Tabla 5

Diferencias en la variable tipo de familia y sexo y la interacción entre ambas, en el aspecto Comportamental

\begin{tabular}{llll}
\hline $\begin{array}{l}\text { Escala } \\
\text { Comportamental }\end{array}$ & Sexo & $\begin{array}{l}\text { Tipo de } \\
\text { Familia }\end{array}$ & Sexo x Tipo Familia \\
\hline Escala SS: Inadecuado Control del Impulso & $\mathrm{F}=2.37$ & $\mathrm{~F}=0.32$ & $\mathrm{~F}=2.15$ \\
$\begin{array}{l}\text { Escala TT: Disconformidad Social } \\
\begin{array}{l}\text { Escala UU: Rendimiento Escolar } \\
\text { Inadecuado }\end{array}\end{array}$ & $\mathbf{F}=\mathbf{1 0 . 9 2 * *}$ & $\mathrm{F}=0.31$ & $\mathbf{F}=\mathbf{5 . 3 3 *}$ \\
Escala WW: Desinterés por la Escuela & $\mathrm{F}=0.74$ & $\mathrm{~F}=0.03$ & $\mathrm{~F}=2.67$ \\
\hline
\end{tabular}

Notas: $\mathrm{F}=$ Prueba ANOVA. ${ }^{*} \mathrm{p}<0.05 .{ }^{*} \mathrm{p}<0.01$ 
Al observar los promedios aritméticos identificamos que los varones alcanzan valores más elevados que las mujeres en Escala Disconformidad Social y Rendimiento Escolar Inadecuado. En consecuencia, se acepta en parte la hipótesis 6 (Véase Tabla 6).

Tabla 6

Estadísticas de las escalas que obtuvieron diferencias significativas en relación al sexo en lo Comportamental

\begin{tabular}{lccccccc}
\hline \multirow{2}{*}{$\begin{array}{l}\text { Escala } \\
\text { Comportamental }\end{array}$} & \multicolumn{2}{c}{$\begin{array}{c}\text { Varones } \\
(\mathbf{n = 1 1 9 )}\end{array}$} & \multicolumn{2}{c}{$\begin{array}{c}\text { Mujeres } \\
(\mathbf{n = 1 3 5 )}\end{array}$} & \multicolumn{2}{c}{$\begin{array}{c}\text { Diferencias } \\
\text { Estandarizadas }\end{array}$} \\
\cline { 2 - 8 } & $\mathrm{M}$ & $\mathrm{DE}$ & $\mathrm{M}$ & $\mathrm{DE}$ & $\mathrm{D}$ & Etiqueta \\
$\begin{array}{l}\text { Escala TT: Disconformidad Social } \\
\begin{array}{l}\text { Escala UU: Rendimiento Escolar } \\
\text { Inadecuado }\end{array}\end{array}$ & $\mathbf{1 2 . 8 6}$ & 4.295 & 11.60 & 4.375 & 0.29 & Pequeña \\
\hline
\end{tabular}

Notas: $\mathrm{D}=$ Diferencia.

A nivel específico al analizar los promedios aritméticos se evidencia que los varones de Familia Monoparental presentan valores más elevados que los adolescentes de Familia Nuclear Biparental y que las adolescentes mujeres de Familia Monoparental en la Escala Disconformidad Social.

Al analizar las diferencias en la variable tipo de familia y sexo y la interacción entre ambas, en el Clima Social Familiar se observa que los adolescentes de familia nuclear biparental y monoparental no se diferencian en el Clima Social Familiar; cuando se toma en cuenta el sexo de los mismos tampoco se hallan diferencias significativas en ninguna de las dimensiones. Con la información del párrafo anterior, en referencia a la hipótesis 7, se acepta la hipótesis nula (Véase Tabla 7).

\section{Tabla 7}

Diferencias en la variable tipo de familia y sexo y la interacción entre ambas, en el Clima Social Familiar

\begin{tabular}{lccc}
\hline $\begin{array}{l}\text { Dimensiones del } \\
\text { Clima Social Familiar }\end{array}$ & Sexo & Tipo de familia & Sexo x Tipo Familia \\
\hline Relación & $\mathrm{F}=0.81$ & $\mathrm{~F}=0.404$ & $\mathrm{~F}=0.005$ \\
Estabilidad & $\mathrm{F}=0.74$ & $\mathrm{~F}=0.40$ & $\mathrm{~F}=0.04$ \\
Desarrollo & $\mathrm{F}=0.04$ & $\mathrm{~F}=0.09$ & $\mathrm{~F}=0.16$ \\
\hline
\end{tabular}

Con la misma información de la Tabla 7, respecto a la hipótesis 8 , se acepta la hipótesis nula. No existe diferencias estadísticas significativas en el Clima Social Familiar (Dimensiones Relación, Estabilidad, Desarrollo) según el sexo de los adolescentes. 


\section{DISCUSIÓN}

Encontramos que el tipo de familia (Nuclear Biparental y Monoparental) no impacta en los Estilos de Personalidad, no obstante, el sexo de los participantes si impacta sobre la personalidad. Los varones tienden a ser menos cooperativos y menos respetuosos y más violentos que las mujeres. Estos datos coinciden con el estudio de Flores (1993), y el de Frisancho (2011) quienes hallaron que los varones utilizan significativamente de modo más intenso la agresión física en comparación con las mujeres, y muestran una tendencia a hacer uso o expresar una actitud de resentimiento.

Esta información se precisa con los análisis de la interacción tipo de familia/ sexo, donde se observa que los varones de familia monoparental tienden a ser más violentos que las mujeres de su propio grupo y que los adolescentes de familia nuclear biparental. Estos hallazgos coinciden con el estudio de Clemens (1988) quien identifica signos de agresión y depresión en jóvenes de familia disgregada, caracterizada por la ausencia de figura paterna o de ambos padres; y también coincide con la investigación de Perales y Sogui (1997) que identificaron como factor de riesgo hacia violencia la edad entre 15 y 17 años, la estructura familiar desintegrada, mayor densidad habitacional, tendencia antisocial, entre otros aspectos. En esta misma línea, Commanor y Phillips (2002) encontraron una fuerte relación estadística entre la ausencia del padre y la delincuencia juvenil y/o violencia. Asimismo, Wilson (1994, en Kliksberg, 2004) reporta que los niños que viven con un solo progenitor son más propensos a tener problemas de conducta, dificultades con sus compañeros y conducta antisocial. Estos resultados pueden ser explicados con los estudios de Chouhy (2001) y Gaddini (1979), quienes señalan que la presencia del padre permite a los hijos la autorregulación de la conducta, introduce un orden interno en el niño, colocando los limites en la relación con la madre y sus congéneres.

Por otro lado, el tipo de familia (Nuclear Biparental y Monoparental) si impacta sobre los Sentimientos y Actitudes, en lo referido a la Inaceptación Sexual, es decir que la composición familiar si influye en actitudes de aceptación y/o de culpabilidad o vergüenza hacia su sexo. Este resultado se corresponde con los aportes de Aberastury y Salas (1984) y Vaeza (2001) quienes enfatizan que el padre es la segunda persona adulta, además de la madre, que ofrece su persona, su cuerpo, sus sentimientos, y que facilita a la estructuración psíquica del hijo, particularmente al logro de una identidad de género y de una identificación sexual.

En otro de los resultados de la interacción tipo de familia/sexo, se observa que los varones que pertenecen a Familia Monoparental perciben un Clima Familiar Inadecuado en relación a los adolescentes de Familia Nuclear Biparental; resultados que guardan relación con el reporte de Bengochea (1996) que encontró diferencias significativas en el clima familiar e insatisfacción familiar entre niños 
de padres separados, huérfanos o de padres desconocidos con los de familia intacta, pero difieren de los resultados de Jiménez, Fernández y Godoy (2000) quienes no encontraron diferencias significativas en el clima familiar entre adolescentes de padres tanto separados como los que permanecen juntos, y también difiere de los estudios de Salazar (2003), que reporta que los tipos de familia no son por sí mismas variables relevantes para influir en las formas de interrelación familiar.

También en relación a los Sentimientos y Actitudes, se encuentra que los varones de Familia Monoparental presentan mayor Desconfianza Escolar, entendida como una desconfianza general en la escuela, hallazgo que está en línea con los estudios de MacLanahan y Sandefur (1994, en Chouhy, 2001) quienes al comparar a jóvenes que crecieron con padre y sin padre observaron que el riesgo a permanecer sin estudiar ni trabajar por periodos prolongados y de interrumpir estudios secundarios es un $50 \%$ más alto en jóvenes que crecieron sin su padre. Asimismo, Wilson (1994, en Kliksberg, 2004) reportó que los menores que viven únicamente con un solo padre, son dos doblemente propensos a ser retirados o suspendidos de la institución educativa, a presentar problemas emocionales y de conducta y a tener problemas de relación con sus compañeros.

Otro aspecto interesante que encontramos es que los varones en general presentan mayor Intolerancia Social que las mujeres, vale decir que tienden a tener un menor respeto por las normas sociales y derechos de los demás. Este hallazgo se expresaría en términos culturales, es decir como una tendencia general presente en los adolescentes varones de Lima.

En relación al aspecto comportamental, el tipo de familia (Nuclear Biparental y Monoparental) no tiene impacto en este aspecto; pero el sexo si impacta sobre lo comportamental. Los varones en general tienden a presentar mayor Rendimiento Escolar Inadecuado que las mujeres; en tanto que el análisis de la interacción sexo/tipo de familia encuentra que los varones de familia monoparental son los que tienen mayor Disconformidad Social. En referencia al Rendimiento Escolar Inadecuado, Paz-Navarro et al. (2009) reportaron que los estudiantes con bajo rendimiento escolar pertenecían a varios tipos de familia (completa, extensa, reconstruida y monoparental) en tanto que, un alto porcentaje de los alumnos con rendimiento escolar promedio pertenecían a familia completa. Al respecto Katz (1980) enfatiza que la desintegración familiar, la situación conflictiva entre los padres, y la mala relación entre el hijo y uno de los padres, puede generar que el adolescente canalice inconscientemente su agresión dejando de funcionar en la escuela; evidenciándose una disminución en la capacidad de aprendizaje o la del aprovechamiento.

Por otro lado, en lo concerniente a la Disconformidad Social, vale decir disconformidad con las normas y ética social, nuestros resultados coinciden con los de Ángel y Ángel (en Chouhy, 2001) quienes señalan que los adolescentes de 
familia monoparental presentan dificultades en el control de sus impulsos, son más vulnerables a la presión de sus coetáneos y a tener problemas con la ley; y los reportes de Whitehead (mencionado por Kliksberg, 2004) quien encontró que en los jóvenes de los Centros de Detención Juvenil de EEUU, más del 70\% pertenecían a familias donde el padre se encontraba ausente; dato que se corrobora con el reporte de Zugasti (2008) que informa que los hijos que crecen sin conservar el contacto y relación con el padre son 20 veces más propensos a tener desórdenes de conducta y a acabar en prisión.

Finalmente, observamos que el tipo de familia no impacta en el Clima Social Familiar, constructo que se sustenta en tres dimensiones: Relación, Desarrollo y Estabilidad. Es plausible suponer que en las familias monoparentales el clima social familiar fuera de menor calidad que en las familias biparentales, pero no es así, este clima es percibido de manera semejante por los adolescentes de ambos tipos de familia. Este resultado coincide con lo hallado por Jiménez, Fernández y Godoy (2000) y Salazar (2003), quienes no hallaron relaciones significativas entre las dimensiones del clima social familiar y los tipos de familia según su composición y los de Rosales y Espinoza (2009) los mismos que reportan que no existe diferencias entre ambas variables; podemos concluir que la diversidad de estructuras en las familias y que independientemente del tipo de familia de la que se forme parte, no son por sí mismas variables relevantes para influir en el clima social familiar.

\section{CONCLUSIONES}

1. Los varones en general tienden a ser menos cooperativos y menos respetuosos y a utilizar más la violencia que las mujeres.

2. Los varones de Familia Monoparental tienden a ser más violentos que las mujeres de su propio grupo y que los adolescentes de Familia Nuclear Biparental.

3. Los adolescentes de Familia Nuclear Biparental y Monoparental se diferencian significativamente en Inaceptación Sexual. Es decir, el tipo de familia influye en la aceptación y/o rechazo del sexo.

4. Los varones de Familia Monoparental tienden a percibir un Clima Familiar Inadecuado en relación a los adolescentes de Familia Nuclear Biparental y las mujeres de su propio grupo.

5. Los varones de Familia Monoparental tienden a presentar mayor Desconfianza Escolar, entendida como una desconfianza general en la escuela. 
6. Los varones en general tienden a mostrar mayor Intolerancia Social que las mujeres, es decir que tienden a tener menos respeto por las normas sociales y derechos de los demás.

7. Los varones en general tienden a presentar mayor Rendimiento Escolar Inadecuado que las mujeres.

8. Los varones de familia monoparental muestran un mayor nivel de disconformidad social que las mujeres de su propio grupo y que los adolescentes de Familia Nuclear Biparental, entiéndase como disconformidad con las normas y ética social.

9. Los adolescentes de Familia Nuclear Biparental y Monoparental no presentan diferencias en el Clima Social Familiar (Relación, Desarrollo, Estabilidad).

\section{REFERENCIAS}

Aberastury, A. y Salas, E. (1984). La paternidad. Buenos Aires: Kargieman.

Bengoechea, P. (1996). Un análisis comparativo de respuestas a la privación parental en niños de padres separados y niños huérfanos en régimen de internado. Revista Psicothema, 8 (3) pp. 597-608.

Billings, AG. \& Moos, RH. (1981). El papel de las respuestas de afrontamiento y recursos sociales en la atenuación de la tensión de los acontecimientos vitales. Journal of Behavioral Medicine. 4, 139-157.

Calderón, C y De la Torre, A. (2005). Relación entre clima social familiar y la Inteligencia Emocional en alumno de $5^{\circ}$ año de secundaria de colegios particulares mixtos de Trujillo. (Tesis de Licenciatura). Universidad César Vallejo, Trujillo, Perú.

Cardenal, V., Sánchez, M ${ }^{\text {a }}$ P., y Ortiz-Tallo, M. (2007). Los trastornos de personalidad según el modelo de Millon: una propuesta integradora. Clínica y Salud, 18. Recuperado de:http://scielo.isciii.es/scielo.php?script=sci_arttext\&pid $=$ S1130-2742007000300004

Clemens, M. (1988). A propósito de la agresión y depresión en un grupo de niños institucionalizados. (Tesis de Licenciatura). Pontificia Universidad Católica del Perú, Lima, Perú.

Commanor, W. \& Phillips, L. (2002). El impacto de los ingresos y la estructura familia en la delincuencia. Revista de Economía Aplicada, 5 (2), pp. 209-232. Recuperado de http://ageconsearch.umn.edu/bitstream/44078/2/comanor.pdf

Chouhy, R. (2000). Función Paterna y familia monoparental. ¿Cuál es el costo de prescindir del padre?. Perspectivas Sistémicas, 66(13). Recuperado de http://www.redsistemica. com.ar 
Flores, E. (1993). Características de la agresividad en estudiantes secundarios de ambos sexos de nivel socio-económico bajo. (Tesis de Licenciatura). Universidad Inca Garcilaso de la Vega, Lima, Perú.

Frisancho, Y. (2011). Violencia Escolar o Bullying relacionado con el Funcionamiento Familiar, adolescentes de la I.E. "Mariscal Cáceres" Tacna - 2011". (Tesis de Licenciatura). Universidad Nacional Jorge Basadre Grohmann, Tacna, Perú.

Gaddini, E. (1979). Formación del padre y Escena primaria. Revista de Psicoanálisis, tomo XXXVI, (1). Recuperado de: file:///C:/Users/user/Downloads/402-1537-1-PB. pdf

Hernández, R., Fernández, C. y Baptista, P. (2010). Metodología de la investigación (11 ${ }^{\mathrm{a}}$ Ed.). México: McGraw-Hill.

Heim, A. \& Westen, D. (2007). Teorías de la personalidad y trastornos de la personalidad. En J. Oldha, A. Skodol, D. Bender. Tratado de los trastornos de la personalidad. (Cap. 2). Barcelona, España: Masson.

Jiménez, T., Fernández, R., y Godoy, F. (2000). Evaluación del clima familiar en una muestra de adolescentes hijos de padres separados. Revista Iberoamericana de Diagnóstico y Evaluación Psicológica, 9 (1) pp. 61-72.

Katz, L. (1980). Mothering and teaching-Some significant distinctions. In L.G. Katz (Ed.), Current topics in early childhood education. 3, pp. 4763. Norwood: Ablex.

Kliksberg, B. (2004). Evolución de la relación del niño, la niña y el adolescente con la Familia. Ponencia presentada en el XIX Congreso Panamericano del Niño, 27-29 octubre; México. Recuperado de: http://www.redalyc.org/pdf/105/10503818.pdf

Moos, R., Moos B. \& Trickett, E. (1984). FES, WES y CES. Escalas de clima social. Madrid, España: TEA Ediciones

Paz-Navarro, L., Rodríguez, P., Martínez, M. (2009). Funcionamiento familiar de alumnos con bajo Rendimiento escolar y su comparación con un grupo de rendimiento promedio en una preparatoria. Revista Virtual Universidad Católica del Norte (40). Recuperado de: http://www.redalyc.org/articulo.oa?id=194229200009

Perales, A. y Sogui, C. (1997). Conductas violentas en adolescentes: identificación de factores de riesgo para el diseño de programa preventivo. Monografía de investigación (3). Instituto Nacional de Salud Mental Honorio Delgado.

Pichardo, C., Fernández de Haro, E. y Amezcua, J. (2002). Importancia del clima social familiar en la adaptación personal y social de los adolescentes. Revista de psicología general y aplicada: Federación Española de Asociaciones de Psicología, 55(4), pp. 575-590. Recuperado de: http:// dialnet.unirioja.es/buscar/documentos?querysDismax.DOCUMENTAL_ $\mathrm{TODO}=$ importancia + del + clima + social + familiar + en + la + adaptacion + personal +

Rosales, C. y Espinoza, M. (2009). La percepción del clima familiar en adolescentes miembros de diferentes tipos de familia. En Psicología y Ciencia Social. 10 (1), p 
64-71. Recuperado de: http://tuxchi.iztacala.unam.mx/ojs/index.php/pycs/article/ viewFile/17/15

Ruiz, C. y Guerra, E. (1993). Estandarización del Test FES /Escala de Clima Social en la familia para Lima Metropolitana. Lima.

Sánchez, H. y Reyes, C. (2006) Metodología y diseños en la investigación científica. Lima: Visión universitaria

Salazar, A. (2003). Relación entre Clima Social Familiar y Tipos de Familia en un grupo de adolescentes gestantes de un Hospital de la Provincia Constitucional del Callao. (Tesis de Licenciada). USMP, Lima, Perú.

Vaeza, R. (2001). “PPaternidad en crisis?”. Recuperado de: http://www.querencia.psico. edu.uy/revista_nro3/rosario_vaeza.htm

Zavala, G. (2001). El Clima Familiar su relación con los intereses vocacionales y los tipos caracterológicos de los alumnos del 5to. Año de Secundaria de los colegios nacionales del Distrito del Rímac. (Tesis Licenciatura). UNMSM, Lima, Perú.

Zugasti, F. (2008). Custodia compartida. Madridpress.com. Diario independiente de Madrid, España. Recuperado de: http://madridpress.com/not/70857/custodia compartida 